https://helda.helsinki.fi

\title{
Partition functions for supersymmetric gauge theories on spheres
}

\author{
Bytsenko, A. A.
}

2018-10-30

Bytsenko , A A , Chaichian , M \& Goncalves , A E 2018 , ' Partition functions for supersymmetric gauge theories on spheres ' , International Journal of Modern Physics A, vol. 33 , no. 30 , 1850179 . https://doi.org/10.1142/S0217751X18501798

http://hdl.handle.net/10138/325816

https://doi.org/10.1142/S0217751X18501798

acceptedVersion

Downloaded from Helda, University of Helsinki institutional repository.

This is an electronic reprint of the original article.

This reprint may differ from the original in pagination and typographic detail.

Please cite the original version. 


\title{
Partition Functions for Supersymmetric Gauge Theories on Spheres
}

\author{
A. A. Bytsenko ${ }^{(a)}{ }^{1}$, M. Chaichian ${ }^{(b)}{ }^{2}$ and A. E. Gonçalves ${ }^{(a)} 3$ \\ (a) Departamento de Física, Universidade Estadual de Londrina \\ Caixa Postal 6001, Londrina-Paraná, Brazil \\ (b) Department of Physics, University of Helsinki \\ P.O. Box 64, FI-00014 Helsinki, Finland
}

\begin{abstract}
In this paper we briefly review the main idea of the localization technique and its extension suitable in supersymmetric gauge field theory. We analyze the partition function of the vector multiplets with supercharges and its blocks on the even- and odddimensional spheres and squashed spheres. We exploit so-called Faà di Bruno's formula and show that multipartite partition functions can be written in the form of expansion series of the Bell polynomials. Applying the restricted specialization argument we show that $q$-infinite-product representation of partition functions admits presentation in terms of the Patterson-Selberg (or the Ruelle-type) spectral functions.
\end{abstract}

PACS: 03.70.+k, 11.10.-z (Quantum field theory); 04.62.+v (Quantum fields in curved spacetime); 11.15.-q (Gauge field theories); 02.30.Gp (Special functions)

Key words and phrases. Localization technique. Partition functions in gauge supersymmetric quantum field theory. The Bell polynomials and spectral functions of hyperbolic geometry.

\footnotetext{
${ }^{1}$ E-mail: aabyts@gmail.com

${ }^{2}$ E-mail: masud.chaichian@helsinki.fi

${ }^{3}$ E-mail: aedsongoncalves@gmail.com
} 


\section{Contents}

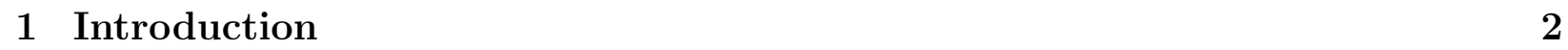

$\begin{array}{lll}2 & \text { Partition functions } & 3\end{array}$

2.1 The partition function of the vector multiplets . . . . . . . . . . . . . 3

$2.2 \quad$ Infinite-product representation for $\Upsilon_{r}(x \mid \epsilon)$ and $S_{r}(x \mid \epsilon)$. . . . . . . . . . . 5

\begin{tabular}{|lrr}
\hline 3 & The Bell polynomials & 6
\end{tabular}

\begin{tabular}{|llr}
\hline & Restricted specializations & 8 \\
\hline
\end{tabular}

4.1 Symmetry and modular properties . . . . . . . . . . . . . . . . . . . . 10

$5 \quad$ Factorization for the partition function of the squashed sphere $\quad 11$

\section{Introduction}

One of the goals of this paper is to give the extension of the localization formula to the path integral in the context of supersymmetric quantum field theory. The localization technique can be applied to certain observables, such as partition functions in supersymmetric gauge theory.

The plan of this paper is as follows. In Section 2 we analyze partition functions for supersymmetric gauge theories on spheres. The partition function of the vector multiplets with supercharges on the even- and odd-dimensional spheres and squashed spheres is reviewed in Section 2.1. Partition functions can be constructed by using the special functions $\Upsilon_{r}(x \mid \epsilon)$ and $S_{r}(x \mid \epsilon)$. The main building block for these functions is the multiple Gamma function $\gamma_{r}(x \mid \epsilon)$.

Then in Section 3 we use the so-called Faà di Bruno's formula and show that multipartite partition functions can be written in the form of expansion series by means of the Bell polynomials.

Restricted specializations we analyze in Section 4. We derive an infinite-product formula for the partition function in the form of the Ruelle spectral functions, whose spectrum is encoded in the Patterson-Selberg functions of the hyperbolic three-geometry. In Section 4.1 we describe the symmetry and modular properties in $q$-infinite-product structure for an appropriate blocks of the partition functions.

Finally in Section 5 we describe the holomorphic block $B_{\alpha}$ associated with the partition function on appropriate supersymmetric background. Then we compute the partition functions in terms of the spectral Ruelle functions and briefly discuss some applications of these computations. 


\section{Partition functions}

Motivations for localization technique in physics. In this paper we are interested in what can be said as a localization in (supersymmetric) gauge field theory. Usually the localization technique applied to supersymmetric observables, such as partition functions, supersymmetric Wilson loops, etc. It is important that the supersymmetric localization gives opportunity to study the nonperturbative results for these class of observables. Also this is a powerful tool for analysis of interacting quantum field theory. The localization answers can be given in terms of complicated finite dimensional integrals. Therefore techniques to study of these integrals and the relevant physical and mathematical information should be deduced and developed.

\subsection{The partition function of the vector multiplets}

Let us consider some examples of the supersymmetric gauge theories on spheres $S^{d}$. The attempt of calculation of the partition function on $S^{d}$ in the case of a continuous range of $d$ has been made in [1]. Useful analysis for localization of equivariant cohomology for compact and non-compact group actions (the Duistermaat-Heckman formula; Harish-Chandra, ItzyksonZuber integral formulas) the reader can find in article [2]. These results were generalized and extended to the squashed spheres. Term squashing in other words means the homogeneous deformation (see also Section 5).

The key principle that can be used for computation of the partition function is the localization argument. The partition function of the vector multiplet with 4, 8 and 16 supercharges placed on the even- and odd-dimensional spheres [3] is given respectively by

$$
\begin{aligned}
Z_{S^{2 r}} & =\int_{\mathfrak{g} \prime} d a \prod_{w \in R_{A d \mathfrak{g}}} \Upsilon_{r}(i w \cdot a \mid \epsilon) e^{P_{r}(a)}+\cdots, \\
Z_{S^{2 r-1}} & =\int_{\mathfrak{g} \prime} d a \prod_{w \in R_{A d \mathfrak{g}}} S_{r}(i w \cdot a \mid \epsilon) e^{P_{r}(a)}+\cdots .
\end{aligned}
$$

In Eqs. 2.1) and (2.2) the polynomial $P_{r}(a)=\sum_{j=1}^{r} \alpha_{j} \operatorname{Tr}\left(a^{j}\right)$ is coming from the classical action of the theory, the integrals are taken over the Cartan subalgebra $\mathfrak{g}$ ' of the gauge Lie algebra $\mathfrak{g}$, the $w$ are weights of adjoint representation of $\mathfrak{g}$. The parameters $\alpha_{j}$ are associated to the Yang-Mills coupling, the Chern-Simons couplings and Fayet-Iliopoulos couplings [4]. $\epsilon$-parameters are equivariant parameters of the $T^{r} \subset S O(2 r)$ toric action on $S^{2 r-1}$ (also $\epsilon_{1}, \ldots, \epsilon_{r}$ are the squashing parameters for $\left.S^{2 r}\right)$. The case of the odd and even dimensional spheres $S^{2 r-1}$ and $S^{2 r}$ leads to two type of special functions called $S_{r}$ and $\Upsilon_{r}$ respectively, that can present the final result for the partition function.

Remark 2.1 The nature of the dots in Eqs. (2.1) and (2.2) for arbitrary $r$ is not well clear. Indeed:

- For supersymmetric gauge theories on $S^{2}$ there is $\mathcal{N}=2$ vector multiplet with 4 supercharges [5, 6] and the dots are nonperturbative contributions coming from localization 
loci with nontrivial magnetic fluxes.

- For $S^{3}$ there is $\mathcal{N}=2$ vector multiplet with 4 supercharges (see Refs. [7] and [8] for a review) and the dots are absent.

- For $S^{4}$ there is $\mathcal{N}=2$ vector multiplet with 8 supercharges [4] and the dots correspond to the contributions of pointlike instantons. These poles can be computed using the Nekrasov instanton partition function [9].

- For $S^{5}$ there is $\mathcal{N}=1$ vector multiplet with 8 supercharges [10, 11] and there are no systematic derivation and understanding of the dots (also for the case $S^{7}$ ).

- For $S^{6}$ there is $\mathcal{N}=2$ vector multiplet with 16 supercharges [1], and the nature of the dots remains to be understood.

- For $S^{7}$ there is $\mathcal{N}=1$ vector multiplet with 16 supercharges [1].

The main building block for functions $S_{r}$ and $\Upsilon_{r}$ is the multiple Gamma function $\gamma_{r}\left(x \mid \epsilon_{1}, \ldots, \epsilon_{r}\right)$, it is a function of a variable $x$ on complex plane $\mathbb{C}$ and $r$ complex parameters $\epsilon_{1}, \ldots, \epsilon_{r}$.

Function $\gamma_{r}\left(x \mid \epsilon_{1}, \ldots, \epsilon_{r}\right)$ can be defined as a zeta-regularized product

$$
\gamma_{r}(x \mid \epsilon)=\gamma_{r}\left(x \mid \epsilon_{1}, \ldots, \epsilon_{r}\right)=\prod_{n_{1}, \ldots, n_{r}=0}^{\infty}\left(x+n_{1} \epsilon_{1}+\cdots+n_{r} \epsilon_{r}\right) .
$$

In Eq. (2.3) the parameters $\epsilon_{j}$ belong to an open half-plane of $\mathbb{C}$ bounded by a real line passing trough the origin. Among other things the unrefined version of $\gamma_{r}$ is defined as

$$
\gamma_{r}(x)=\gamma_{r}(x \mid 1, \ldots, 1)=\prod_{k=0}^{\infty}(x+k)^{\frac{(k+1)(k+2) \cdots(k+r-1)}{(r-1) !}} .
$$

The localization on $S^{2 r}$ based on the $\Upsilon_{r}$-function, which can be defined as

$$
\Upsilon_{r}(x \mid \epsilon)=\Upsilon_{r}\left(x \mid \epsilon_{1}, \ldots, \epsilon_{r}\right)=\gamma_{r}\left(x \mid \epsilon_{1}, \ldots \epsilon_{r}\right) \gamma_{r}\left(\sum_{j=1}^{r} \epsilon_{j}-x \mid \epsilon_{1}, \ldots, \epsilon_{r}\right)^{(-1)^{r}}
$$

The unrefined version of $\Upsilon_{r}$ can be defined as follows

$$
\Upsilon_{r}(x)=\Upsilon_{r}(x \mid 1, \ldots, 1)=\prod_{k \in \mathbb{Z}}(k+x)^{\operatorname{sgn}(k+1) \frac{(k+1)(k+2) \cdots(k+r-1)}{(r-1) !}} .
$$

The $S_{r}$-function, as defined below (see Ref. [12] for details), plays an important role upon localization on $S^{2 r-1}$ :

$$
S_{r}(x \mid \epsilon)=S_{r}\left(x \mid \epsilon_{1}, \ldots, \epsilon_{r}\right)=\gamma_{r}\left(x \mid \epsilon_{1}, \ldots, \epsilon_{r}\right) \gamma_{r}\left(\sum_{j=1}^{r} \epsilon_{j}-x \mid \epsilon_{1}, \ldots, \epsilon_{r}\right)^{(-1)^{r-1}}
$$




\subsection{Infinite-product representation for $\Upsilon_{r}(x \mid \epsilon)$ and $S_{r}(x \mid \epsilon)$}

By definition the infinite-product of the $\Upsilon_{r}(x \mid \epsilon)$ has the form [3]:

$$
\Upsilon_{r}(x \mid \epsilon) \stackrel{\text { reg }}{=} \prod_{n_{1}=0, \ldots, n_{r}=0}^{\infty}\left(x+\sum_{s=1}^{r} n_{s} \epsilon_{s}\right)\left(\epsilon-x+\sum_{s=1}^{r} n_{s} \epsilon_{s}\right)^{(-1)^{r}}
$$

In Eq. 2.8) $\stackrel{\text { reg }}{=}$ denotes zeta-function regularization. Also the infinite-product definition for the $S_{r}(x \mid \epsilon)$ is [3]:

$$
S_{r}(x \mid \epsilon) \stackrel{\text { reg }}{=} \prod_{n_{1}=0, \ldots, n_{r}=0}^{\infty}\left(x+\sum_{s=1}^{r} n_{s} \epsilon_{s}\right)\left(\epsilon-x+\sum_{s=1}^{r} n_{s} \epsilon_{s}\right)^{(-1)^{r-1}} .
$$

$\Upsilon_{r}$-function 2.8 and $S_{r}$-function 2.9 can be represented in the following forms

$$
\begin{aligned}
H_{r}(x \mid \epsilon) & =\mathfrak{A}_{r}(x \mid \epsilon) \cdot \mathfrak{B}_{r}(x \mid \epsilon)^{(-1)^{r}} \\
G_{r}(x \mid \epsilon) & =\mathfrak{A}_{r}(x \mid \epsilon) \cdot \mathfrak{B}_{r}(x \mid \epsilon)^{(-1)^{r-1}}
\end{aligned}
$$

where

$$
\begin{aligned}
\mathfrak{A}_{r}(x \mid \epsilon) & =\prod_{n_{1}=0, \ldots, n_{r}=0}^{\infty}\left(1-e^{2 \pi i x} e^{2 \pi \vec{n} \vec{\epsilon}}\right), \\
\mathfrak{B}_{r}(x \mid \epsilon) & =\prod_{n_{1}=0, \ldots, n_{r}=0}^{\infty}\left(1-e^{2 \pi i\left(\sum_{j=1}^{r} \epsilon_{j}-x\right)} e^{2 \pi i \vec{n} \vec{\epsilon}}\right) .
\end{aligned}
$$

Remark 2.2 On the time being our discussions can be summarized as follows:

$\Upsilon_{r}$-functions form a hierarchy with respect to a shift of $x$ by one of $\epsilon$-parameters

$$
\Upsilon_{r}\left(x+\epsilon_{j} \mid \epsilon_{1}, \ldots, \epsilon_{j}, \ldots, \epsilon_{r}\right)=\Upsilon_{r-1}^{-1}\left(x \mid \epsilon_{1}, \ldots, \epsilon_{j-1}, \epsilon_{j+1}, \ldots \epsilon_{r}\right) \Upsilon_{r}\left(x \mid \epsilon_{1}, \ldots, \epsilon_{j}, \ldots, \epsilon_{r}\right) .
$$

$S_{r}$-functions make up a hierarchy with respect to a shift of $x$ by one of the $\epsilon$-parameters

$$
S_{r}\left(x+\epsilon_{j} \mid \epsilon_{1}, \ldots, \epsilon_{j}, \ldots, \epsilon_{r}\right)=S_{r-1}^{-1}\left(x \mid \epsilon_{1}, \ldots, \epsilon_{j-1}, \epsilon_{j+1}, \ldots, \epsilon_{r}\right) S_{r}\left(x \mid \epsilon_{1}, \ldots, \epsilon_{j}, \ldots, \epsilon_{r}\right) .
$$

Expression for $\mathfrak{B}_{r}(x \mid \epsilon)$ can be obtained from $\mathfrak{A}_{r}(x \mid \epsilon)$ by shifting of $x$ by $\epsilon$-parameters: $\mathfrak{B}_{r}(x \mid \epsilon)=$ $\mathfrak{A}_{r}\left(x \mapsto \sum_{j=1}^{r} \epsilon_{j}-x \mid \epsilon\right)$.

$$
\begin{aligned}
& H_{r(\text { odd } r)}(x \mid \epsilon)=\frac{\mathfrak{A}_{r}(x \mid \epsilon)}{\mathfrak{B}_{r}(x \mid \epsilon)}, \quad H_{r(\text { even } r)}(x \mid \epsilon)=\mathfrak{A}_{r}(x \mid \epsilon) \cdot \mathfrak{B}_{r}(x \mid \epsilon), \\
& G_{r(\text { even } r)}(x \mid \epsilon)=\frac{\mathfrak{A}_{r}(x \mid \epsilon)}{\mathfrak{B}_{r}(x \mid \epsilon)}, \quad G_{r(\text { odd } r)}(x \mid \epsilon)=\mathfrak{A}_{r}(x \mid \epsilon) \cdot \mathfrak{B}_{r}(x \mid \epsilon) .
\end{aligned}
$$




\section{The Bell polynomials}

Let us consider for any ordered $r$-tuple of nonnegative integers (not all zeros) $\epsilon=\left(\epsilon_{1}, \epsilon_{2}, \ldots, \epsilon_{r}\right.$ ) the (multi)partitions, i.e. distinct representations of $\left(\epsilon_{1}, \epsilon_{2}, \ldots, \epsilon_{r}\right)$ as sums of multipartite numbers. Because of Eq. (2.12) we have

$$
\begin{aligned}
\log \mathfrak{A}_{r}(x \mid \epsilon) & =\sum_{n_{1}=0, \ldots, n_{r}=0}^{\infty} \log \left(1-e^{2 \pi i x+2 \pi i \vec{n} \vec{\epsilon}}\right) \\
& =-\sum_{n_{1}=0, \ldots, n_{r}=0}^{\infty} \sum_{m=1}^{\infty} \frac{e^{2 \pi i x m}}{m} e^{2 \pi i n_{1} \epsilon_{1} m} \cdots e^{2 \pi i n_{r} \epsilon_{r} m} \\
& =-\sum_{m=1}^{\infty} \frac{e^{2 \pi i x m}}{m}\left(1-e^{2 \pi i \epsilon_{1} m}\right)^{-1} \cdots\left(1-e^{2 \pi i \epsilon_{r} m}\right)^{-1} \\
& =-\sum_{m=1}^{\infty} \frac{e^{2 \pi i x m}}{m} \prod_{j=1}^{r}\left(1-e^{2 \pi i \epsilon_{j} m}\right)^{-1} .
\end{aligned}
$$

Let $\psi(m \mid r):=\prod_{j=1}^{r}\left(1-e^{2 \pi i \epsilon_{j} m}\right)^{-1}$, then

$$
\begin{aligned}
\mathfrak{A}_{r}(x \mid \epsilon) & =\exp \left(-\sum_{m=1}^{\infty} \frac{e^{2 \pi i x m}}{m} \psi(m \mid r)\right) \\
\mathfrak{B}_{r}(x \mid \epsilon) & =\exp \left(-\sum_{m=1}^{\infty} \frac{e^{2 \pi i\left(\sum_{j=1}^{r} \epsilon_{j}-x\right) m}}{m} \psi(m \mid r)\right)
\end{aligned}
$$

Let us introduce the following series expansions:

$$
\begin{aligned}
\mathfrak{A}_{r}(x \mid \epsilon) & =1+\sum_{m=1}^{\infty} \mathcal{C}_{m} e^{2 \pi i x m} \\
\mathfrak{B}_{r}(x \mid \epsilon) & =1+\sum_{m=1}^{\infty} \mathcal{C}_{m} e^{2 \pi i\left(\sum_{j=1}^{r} \epsilon_{j}-x\right) m} .
\end{aligned}
$$

The Bell polynomials are very useful in many problems in combinatorics, specially in view of extensive tables of Bell polynomials. We would like to note their application for the multipartite partition problem [13]. In particular the Bell polynomials technique can be used for calculation of $\mathcal{C}_{m}$.

The Bell polynomials, first extensively studied by E. T. Bell [14], arise in the task of taking the $n$-th derivative of a composite function $h(t)=f(g(t))$. We can find a formula for the $n$-th derivative of $h(t)$. Indeed, let us denote $d^{n} h / d t^{n}=h_{n}, d^{n} f / d g^{n}=f_{n}$ and $d^{n} g / d t^{n}=g_{n}$, then we have $h_{1}=f_{1}, h_{2}=f_{1} g_{2}+f_{2} g_{1}^{2}, h_{3}=f_{1} g_{3}+3 f_{2} g_{2} g_{1}+f_{3} g_{1}^{3}, \ldots$ By mathematical induction we find $h_{n}=f_{1} \alpha_{n 1}\left(g_{1}, \ldots, g_{n}\right)+f_{2} \alpha_{n 2}\left(g_{1}, \ldots, g_{n}\right)+\cdots+f_{n} \alpha_{n n}\left(g_{1}, \ldots, g_{n}\right)$, where $\alpha_{n j}\left(g_{1}, \ldots, g_{n}\right)$ is a homogeneous polynomial of degree $j$ in $g_{1}, \ldots, g_{n}$. As a result, the study of $h_{n}$ may be reduced to the study of the Bell polynomials: $Y_{n}\left(g_{1}, g_{2}, \ldots, g_{n}\right)=\alpha_{n 1}\left(g_{1}, \ldots, g_{n}\right)+$ 
$\alpha_{n 2}\left(g_{1}, \ldots, g_{n}\right)+\cdots+\alpha_{n n}\left(g_{1}, \ldots, g_{n}\right)$. We should stress that $Y_{n}$ is a polynomial in $n$ variables and the fact that $g_{j}$ was originally an $j$-th derivative is not necessary in the consideration.

Useful expressions for the recurrence relations of the Bell polynomials $Y_{n}\left(g_{1}, g_{2}, \ldots, g_{n}\right)$ and it generating function $\mathrm{B}(z)$ have the forms [13]:

$$
\begin{aligned}
& Y_{n+1}\left(g_{1}, g_{2}, \ldots, g_{k+1}\right)=\sum_{k=0}^{n}\left(\begin{array}{l}
n \\
k
\end{array}\right) Y_{n-k}\left(g_{1}, g_{2}, \ldots, g_{n-k}\right) g_{k+1}, \\
& \mathrm{~B}(z)=\sum_{n=0}^{\infty} Y_{n} z^{n} / n ! \Longrightarrow \log \mathrm{B}(z)=\sum_{n=1}^{\infty} g_{n} z^{n} / n !
\end{aligned}
$$

In order to verify the last formula (3.7) we need to differentiate it with respect to $z$ and observe that a comparison of the coefficients of $z^{n}$ in the resulting equation produces an identity equivalent to 3.6 .

Recall that a partition of a positive integer $\mathrm{n}$ is a finite nonincreasing sequence of positive integers $k_{1}, k_{2}, \ldots, k_{r}$ such that $\sum_{j=1}^{r} k_{j}=n$. The $k_{j}$ are called the parts of the partition. The partition $\left(k_{1}, k_{2}, \ldots, k_{r}\right)$ will be denoted by $\mathbf{k}$, and we shall write $\mathbf{k} \vdash n$ to denote $\mathbf{k}$ is a partition of $n$.

From Eq. (3.6) one can obtain the following explicit formula for the Bell polynomials (it is known as the Faá di Bruno's formula)

$$
Y_{n}\left(g_{1}, g_{2}, \ldots, g_{n}\right)=\sum_{\mathbf{k} \vdash n} \frac{n !}{k_{1} ! \cdots k_{n} !} \prod_{j=1}^{n}\left(\frac{g_{j}}{j !}\right)^{k_{j}}
$$

Finally, the following result holds (see also Ref. [13], Theorem 12.3)

$$
\mathcal{C}_{m}=\frac{1}{m !} Y_{m}(0 ! \psi(1 \mid r), 1 ! \psi(2 \mid r), \ldots,(m-1) ! \psi(m \mid r))
$$

and

$$
\begin{aligned}
\mathfrak{A}_{r}(x \mid \epsilon) & =1+\sum_{m=1}^{\infty} \mathcal{C}_{m} e^{2 \pi i x m} \\
& =1+\sum_{m=1}^{\infty} \frac{1}{m !} Y_{m}(0 ! \psi(1 \mid r), 1 ! \psi(2 \mid r), \ldots,(m-1) ! \psi(m \mid r)) e^{2 \pi i x m} \\
\mathfrak{B}_{r}(x \mid \epsilon) & =1+\sum_{m=1}^{\infty} \mathcal{C}_{m} e^{2 \pi i\left(\sum_{j=1}^{r} \epsilon_{j}-x\right) m} \\
& =1+\sum_{m=1}^{\infty} \frac{1}{m !} Y_{m}(0 ! \psi(1 \mid r), 1 ! \psi(2 \mid r), \ldots,(m-1) ! \psi(m \mid r)) \\
& \times e^{2 \pi i\left(\sum_{j=1}^{r} \epsilon_{j}-x\right) m} .
\end{aligned}
$$


As an example, let us calculate $\mathcal{C}_{2}$ coefficient:

$$
\begin{aligned}
2 \mathcal{C}_{2} & =Y_{2}(\psi(1 \mid r), \psi(2 \mid r))=\psi(1 \mid r)^{2}+\psi(2 \mid r)=\prod_{j=1}^{r}\left(1-e^{2 \pi i \epsilon_{j}}\right)^{-2} \\
& +\prod_{j=1}^{r}\left(1-e^{4 \pi i \epsilon_{j}}\right)^{-1} .
\end{aligned}
$$

\section{Restricted specializations}

For some specializations, when $\left\{\epsilon_{1}, \epsilon_{2}, \ldots, \epsilon_{r}\right\}=\underbrace{\left\{i \vartheta, i \zeta_{2}, i \zeta_{3}, \ldots, i \zeta_{r}\right\}}_{r}$ and $q:=e^{2 \pi i \vartheta}$ we get

$$
\begin{aligned}
\mathfrak{A}_{r}(x \mid \epsilon) & =\prod_{n_{1}=0, \ldots, n_{r}=0}^{\infty}\left(1-e^{2 \pi i x} q^{n_{1}+n_{2} \zeta_{2} / \vartheta+\cdots+n_{r} \zeta_{r} / \vartheta}\right), \\
\mathfrak{B}_{r}(x \mid \epsilon) & =\mathfrak{A}_{r}\left(x \longmapsto i \vartheta+i \sum_{j=2}^{r} \zeta_{j}-x \mid i \vartheta, i \zeta_{2}, \ldots, i \zeta_{r}\right) .
\end{aligned}
$$

Spectral functions of hyperbolic three-geometry. Let us begin by explaining some results on the Patterson-Selberg (the Ruelle type) spectral functions. For details we refer the reader to [15, 16] where spectral functions of hyperbolic three-geometry were considered in connection with three-dimensional Euclidean black holes, pure supergravity, and string amplitudes.

Let $\Gamma^{\gamma} \in G=S L(2, \mathbb{C})$ be the discrete group $\bigsqcup^{4} \Gamma^{\gamma}$ is defined by

$$
\begin{aligned}
\Gamma^{\gamma} & =\left\{\operatorname{diag}\left(e^{2 n \pi(\operatorname{Im} \vartheta+i \operatorname{Re} \vartheta)}, e^{-2 n \pi(\operatorname{Im} \vartheta+i \operatorname{Re} \vartheta)}\right): n \in \mathbb{Z}\right\}=\left\{\gamma^{n}: n \in \mathbb{Z}\right\}, \\
\gamma & =\operatorname{diag}\left(e^{2 \pi(\operatorname{Im} \vartheta+i \operatorname{Re} \vartheta)}, e^{-2 \pi(\operatorname{Im} \vartheta+i \operatorname{Re} \vartheta)}\right) .
\end{aligned}
$$

We may construct a zeta function of Selberg-type for the group $\Gamma^{\gamma} \equiv \Gamma_{(\alpha, \beta)}^{\gamma}$ generated by a single hyperbolic element of the form $\gamma_{(\alpha, \beta)}=\operatorname{diag}\left(e^{z}, e^{-z}\right)$, where $z=\alpha+i \beta$ for $\alpha, \beta>0$. Actually $\alpha=2 \pi \operatorname{Im} \vartheta$ and $\beta=2 \pi \operatorname{Re} \vartheta$. The Patterson-Selberg spectral function $Z_{\Gamma^{\gamma}}(s)$ for Re $s>0$ can be attached to $H^{3} / \Gamma^{\gamma}$ as follows:

$$
Z_{\Gamma^{\gamma}}(s):=\prod_{k_{1}, k_{2} \geq 0}\left[1-\left(e^{i \beta}\right)^{k_{1}}\left(e^{-i \beta}\right)^{k_{2}} e^{-\left(k_{1}+k_{2}+s\right) \alpha}\right] .
$$

The zeros of $Z_{\Gamma^{\gamma}}(s)$ are precisely the set of complex numbers $\zeta_{n, k_{1}, k_{2}}=-\left(k_{1}+k_{2}\right)+$ $i\left(k_{1}-k_{2}\right) \beta / \alpha+2 \pi i n / \alpha$, with $n \in \mathbb{Z}$. The magnitude of the zeta-function is bounded for both $\operatorname{Re} s \geq 0$ and $\operatorname{Re} s \leq 0$, and its growth can be estimated as

$$
\left|Z_{\Gamma^{\gamma}}(s)\right| \leq\left(\prod_{k_{1}+k_{2} \leq|s|} \mathrm{e}^{|s| \ell}\right)\left(\prod_{k_{1}+k_{2} \geq|s|}\left(1-\mathrm{e}^{\left(|s|-k_{1}-k_{2}\right) \ell}\right)\right) \leq C_{1} \mathrm{e}^{C_{2}|s|^{3}}
$$

\footnotetext{
${ }^{4}$ From the point of view of the applications, homologies associated with algebras $\mathfrak{g}=\mathfrak{s l}(N ; \mathbb{C})$ important since they constitute the thechnical basis of the proof of the combinatorial identities of Euler-Gauss-JacobiMacDonald [17.
} 
for suitable constants $\ell, C_{1}, C_{2}$. The first product on the right-hand side of (4.5) gives the exponential growth, while the second product is bounded. The spectral function $Z_{\Gamma \gamma}(s)$ is an entire function of order three and of finite type which can be written as a Hadamard product [16]

$$
Z_{\Gamma^{\gamma}}(s)=\mathrm{e}^{Q(s)} \prod_{\zeta \in \Sigma}\left(1-\frac{s}{\zeta}\right) \exp \left(\frac{s}{\zeta}+\frac{s^{2}}{2 \zeta^{2}}+\frac{s^{3}}{3 \zeta^{3}}\right),
$$

where $\Sigma$ is the set of zeroes $\zeta:=\zeta_{n, k_{1}, k_{2}}$ and $Q(s)$ is a polynomial of degree at most three. (The product formula for entire function (4.6) is known as Weierstrass formula (1876).)

For the next step let us introduce the Ruelle spectral function $\mathcal{R}(s)$ associated with hyperbolic three-geometry [15, 16]. The function $\mathcal{R}(s)$ is an alternating product of more complicate factors, each of which is so-called Patterson-Selberg zeta-functions $Z_{\Gamma^{\gamma}}$. Functions $\mathcal{R}(s)$ can be continued meromorphically to the entire complex plane $\mathbb{C}$, poles of $\mathcal{R}(s)$ correspond to zeros of $Z_{\Gamma^{\gamma}}(s)$.

$$
\begin{aligned}
\prod_{n=\ell}^{\infty}\left(1-q^{a n+\varepsilon}\right) & =\prod_{p=0,1} Z_{\Gamma^{\gamma}}(\underbrace{(a \ell+\varepsilon)(1-i \varrho(\vartheta))+1-a}_{s}+a(1+i \varrho(\vartheta) p)^{(-1)^{p}} \\
& =\mathcal{R}(s=(a \ell+\varepsilon)(1-i \varrho(\vartheta))+1-a),
\end{aligned}
$$

being $\varrho(\vartheta)=\operatorname{Re} \vartheta / \operatorname{Im} \vartheta, \sigma(\vartheta)=(2 \operatorname{Im} \vartheta)^{-1}, a$ is a real number, $\varepsilon, b \in \mathbb{C}, \ell \in \mathbb{Z}_{+}$.

Later on we will use the following notation: $(a)_{n}=a(a+1) \cdots(a+n-1)$ and

$$
\begin{aligned}
(a ; q)_{n}= & \frac{(a ; q)_{\infty}}{\left(a q^{n} ; q\right)_{\infty}}=\prod_{m=0}^{\infty}\left[\left(1-a q^{m}\right) /\left(1-a q^{m+n}\right)\right]=(1-a)(1-a q) \cdots\left(1-a q^{n-1}\right) \\
& n=1,2, \ldots,
\end{aligned}
$$

$n$ is a non-negative integer. The shifted $q$-factorial for $n=0$ is $(a ; q)_{n}=1.5$

Setting $e^{2 \pi i x} q^{n_{1}+n_{2} \zeta_{2} / \vartheta+\cdots+n_{r} \zeta_{r} / \vartheta}=q^{x / \vartheta} \Omega_{r-1} q^{n_{1}}$ with $\Omega_{r-1}=q^{n_{2} \zeta_{2} / \vartheta+\cdots+n_{r} \zeta_{r} / \vartheta}$, we get

$$
\begin{aligned}
\left(q^{x / \vartheta} \Omega_{r-1} ; q\right)_{\infty} & :=\prod_{n_{1}=0}^{\infty}\left(1-\Omega_{r-1} q^{n_{1}+x / \vartheta}\right) \\
& =\mathcal{R}\left(s=\left(x / \vartheta+n_{2} \zeta_{2} / \vartheta+\cdots+n_{r} \zeta_{r} / \vartheta\right)(1-i \varrho(\vartheta))\right) .
\end{aligned}
$$

Therefore the infinite products can be factorized as

$$
\begin{aligned}
& \prod_{n_{2}=0}^{\infty} \prod_{n_{3}=0}^{\infty} \cdots \prod_{n_{r}=0}^{\infty} \prod_{n_{1}=0}^{\infty}\left(1-\Omega_{r-1} q^{n_{1}+x / \vartheta}\right) \\
= & \prod_{n_{2}=0}^{\infty} \prod_{n_{3}=0}^{\infty} \cdots \prod_{n_{r}=0}^{\infty} \mathcal{R}\left(s=\left(x / \vartheta+n_{2} \zeta_{2} / \vartheta+n_{3} \zeta_{3} / \vartheta+\cdots+n_{r} \zeta_{r} / \vartheta\right)(1-i \varrho(\vartheta))\right) .
\end{aligned}
$$

\footnotetext{
${ }^{5}$ The following series expansion holds [18]: $(q ; q)_{\infty}^{-a_{n}}=\prod_{n=0}^{\infty}\left(1-q^{n+1}\right)^{-a_{n}}=1+\sum_{n=1}^{\infty} b_{n} q^{n}$, where $a_{n}$ and $b_{n}$ are integers. Then $n b_{n}=\sum_{j=1}^{n} A_{j} b_{n-j}$, where $A_{j}=\sum_{d \mid j} d a_{d}$. If either sequence $a_{n}$ or $b_{n}$ is given the other $A_{j}$ is uniquely determined.
} 
Using Eq. 2.12) we have

$$
\begin{aligned}
\mathfrak{A}_{r}(x \mid \epsilon) & =\prod_{n_{2}=0, \ldots, n_{r}=0}^{\infty} \prod_{n_{1}=0}^{\infty}\left(1-q^{x / \vartheta+n_{1}+n_{2} \zeta_{2} / \vartheta+\cdots+n_{r} \zeta_{r} / \vartheta}\right) \\
& =\prod_{n_{2}=0, \ldots, n_{r}=0}^{\infty} \mathcal{R}\left(s=\left(x / \vartheta+n_{2} \zeta_{2} / \vartheta+\ldots+n_{r} \zeta_{r} / \vartheta\right)(1-i \varrho(\vartheta))\right) .
\end{aligned}
$$

\subsection{Symmetry and modular properties}

The next step of the iterative loop becomes the Jackson (convergent) double infinite product $\left(q^{x / \vartheta} ; q, t\right)_{\infty}[19]$, where $q=e^{2 \pi i \vartheta}, t=e^{2 \pi i \tau}$,

$$
\begin{aligned}
\left(q^{x / \vartheta} ; q, t\right)_{\infty} & =\prod_{n_{1}=0}^{\infty} \prod_{n_{2}=0}^{\infty}\left(1-q^{x / \vartheta+n_{1}+(\tau / \vartheta) n_{2}}\right) \\
& =\prod_{n_{2}=0}^{\infty} \mathcal{R}\left(s=\left(x / \vartheta+(\tau / \vartheta) n_{2}\right)(1-i \varrho(\vartheta))\right)
\end{aligned}
$$

For the product (4.12) two first order $q$ - and $t$-equations take the forms (see also [20])

$$
\begin{aligned}
& \frac{\left(q^{x / \vartheta} ; q, t\right)_{\infty}}{\left(q q^{x / \vartheta} ; q, t\right)_{\infty}}=\left(q^{x / \vartheta} ; t\right)_{\infty}=\mathcal{R}(s=x / \vartheta(1-i \varrho(\vartheta))+1-\tau / \vartheta) \\
& \frac{\left(q^{x / \vartheta} ; q, t\right)_{\infty}}{\left(t q^{x / \vartheta} ; q, t\right)_{\infty}}=\left(q^{v / \vartheta} ; q\right)_{\infty}=\mathcal{R}(s=x / \vartheta(1-i \varrho(\vartheta))+1)
\end{aligned}
$$

Symmetry properties of Jackson double infinite product $\left(q^{x / \vartheta} ; q, t\right)$ analogous to (modular) properties of the standard elliptic gamma functions. For $z \in \mathbb{C}^{*}$ the order one $\Gamma_{1}$ and double (i.e., the order two) $\Gamma_{2}$ standard elliptic gamma functions have the forms

$$
\begin{aligned}
\Gamma_{1}(z ; q, t) & =\prod_{n_{1}, n_{2}=0}^{\infty}\left(\frac{1-z^{-1} q^{n_{1}+1} t^{n_{2}+1}}{1-z q^{n_{1}} t^{n_{2}}}\right), \\
\Gamma_{2}(z ; q, t, v) & =\prod_{n_{1}, n_{2}, n_{3}=0}^{\infty}\left(1-z^{-1} q^{n_{1}+1} t^{n_{2}+1} v^{n_{3}+1}\right)\left(1-z q^{n_{1}} t^{n_{2}} v^{n_{3}}\right) .
\end{aligned}
$$

The double elliptic gamma function $\Gamma_{2}$ has the following interesting modular properties [21]:

$$
\begin{aligned}
\Gamma_{2}(z ; a, b, c) & =\Gamma_{2}(z / a ;-1 / a, b / a, c / a) \cdot \Gamma_{2}(z / b ; a / b,-1 / b, c / b) \cdot \Gamma_{2}(z / c ; a / c, b / c,-1 / c) \\
& \times \exp \left(\frac{i \pi}{12} B_{44}(z ; a, b, c)\right)
\end{aligned}
$$

where $B_{44}$ is given by

$$
B_{44}(z ; a, b, c)=\lim _{x \rightarrow 0} \frac{d^{4}}{d x^{4}} \frac{x^{4} e^{z x}}{\left(e^{a x}-1\right)\left(e^{b x}-1\right)\left(e^{c x}-1\right)} .
$$




\section{$5 \quad$ Factorization for the partition function of the squas- hed sphere}

In this section we describe an interesting factorization property and holomorphic blocks for the partition functions of the squashed 3-sphere. ${ }^{6}$

For the squashed sphere partition function it was observed in [22] that the partition function can be written as:

$$
Z_{S_{b}^{3}}\left(m_{a}\right)=\sum_{\alpha} B_{\alpha}\left(x_{a} ; q\right) \tilde{B}_{\alpha}\left(\tilde{x}_{a} ; \tilde{q}\right)
$$

In Eq. (5.1) the index $\alpha$ labels vacua of the mass-deformed theory, and $B_{\alpha}$ (respectively, $\left.\tilde{B}_{\alpha}\right)$ are certain holomorphic functions of $q=e^{2 \pi i b^{2}}$ (respectively, $\tilde{q}=e^{2 \pi i b^{-2}}$ ). $x_{a}=e^{2 \pi b m_{a}}$ and $\tilde{x}_{a}=e^{2 \pi b^{-1} m_{a}}$.

Remark 5.1 Similar factorization was conjectured for the supersymmetric index [23], has been described in a unified framework in [24]. Similarly factorize was also shown for the lens space partition function [25, 26] and also for the topological twisted index [26].

In all mentioned cases, the partition functions of a given theory on any of these manifolds are built out of the same objects $B_{\alpha}$, the so-called holomorphic blocks. One of the interesting observation combined with the corresponding spaces of partition functions: $S^{3}, S^{3} / \mathbb{Z}_{p}$ and $S^{2} \times S^{1}$ (see [8] for detail); these spaces admit a Heegard decomposition as a union of two solid tori, $S^{1} \times D^{2}[27$.

In order to make the connection to the partition functions we can use the following relations [8]: $\tilde{q}=g \cdot q, \tilde{x}=g \cdot x$, and $g$ implements the action of the diffeomorphism, acting as: $g=\left(\begin{array}{ll}a & b \\ c & d\end{array}\right) \in S L(2, \mathbb{Z}) \Longrightarrow q=e^{2 \pi i \vartheta} \rightarrow \tilde{q}=e^{ \pm 2 \pi i \varphi(a, b \mid c, d ; \vartheta)}$, where $\varphi(a, b \mid c, d ; \vartheta)=\frac{a \vartheta+b}{c \vartheta+d}, x=$ $e^{2 \pi i \mu} \rightarrow \tilde{x}=e^{ \pm 2 \pi i \varphi(0, \mu \mid c, d ; \vartheta)}$. In the case of a free chiral multiplet charged under a $U(1)$ flavor symmetry, a single block is given by [8]:

$$
B_{\triangle}(x ; q)=\left(q x^{-1} ; q\right)_{\infty}=\prod_{n=0}^{\infty}\left(1-q^{n+1} x^{-1}\right) \stackrel{E q \cdot 4.7]}{=} \mathcal{R}(s=(1-\mu / \vartheta)(1-i \varrho(\vartheta))), \quad|q|<1 .
$$

At the same time

$$
B_{\triangle}(x ; q)=\left(q x^{-1} ; q\right)_{\infty}=\prod_{n=0}^{\infty}\left(1-q^{-n} x^{-1}\right)^{-1}=\mathcal{R}\left(s=-\mu / \vartheta(1-i \varrho(\vartheta)+2)^{-1}, \quad|q|>1,\right.
$$

where symbol $\triangle$ is connected to the Cartan generator of the $S U(2)$ R-symmetry factor.

\footnotetext{
${ }^{6}$ A simple example to construct supersymmetry-preserving geometries which are topologically the ellipsoid and which is a deformation of the three-sphere, preserving a $U(1) \times U(1)$ isometry, can be parametrised as [22]: $b^{2}\left|z_{1}\right|+\left(1 / b^{2}\right)\left|z_{2}\right|^{2}=1, z_{1}, z_{2} \in \mathbb{C}$, where $b$ is the squashing parameter.
} 
The case of the S-fusing gives the $S_{b}^{3}$ partition function (see Eq. (5.1)). Simplify calculations for this case and taking $c=0(a d=1)$ and $b^{2}$ to have positive imaginary part, so that $|q|,|\tilde{q}|^{-1}<1$, we get:

$$
B_{\triangle}(x ; q) B_{\triangle}(\tilde{x} ; \tilde{q})=\prod_{n=0}^{\infty} \frac{1-q^{n+1} x^{-1}}{1-\tilde{q}^{n} \tilde{x}^{-1}}=\frac{\mathcal{R}(s=(1-\mu / \vartheta)(1-i \varrho(\vartheta)))}{\mathcal{R}(s=( \pm b / d \vartheta \mp \mu / d \vartheta)(1-i \varrho(\vartheta))+1 \mp a / d)} .
$$

This reproduce the $S_{b}^{3}$ partition function of a free chiral multiplet with the chosen contact terms.

Note that a similar result occurs for the other partition functions in a gauge field theory where are usually many blocks, and a contour integral prescription for computing them was given in [24]. In addition the blocks were derived directly by localization theorem in [27].

\section{Acknowledgments}

We are much grateful to Markku Oksanen for several remarks and improvements in the work. AAB would like to acknowledge the Conselho Nacional de Desenvolvimento Científico e Tecnológico (CNPq, Brazil) and Coordenacão de Aperfeiçamento de Pessoal de Nível Superior (CAPES, Brazil) for financial support.

\section{References}

[1] J. A. Minahan, Localizing gauge theories on $S^{d}$, JHEP 04 (2016) 152; [arXiv:1512.06924v2 [hep-th]].

[2] A. A. Bytsenko, M. Libine, and F. L. Williams, Localization of Equivariant Cohomology for Compact and Non-Compact Group Actions, Journal of Dynamical Systems and Geometric Theories, 3 (2005) 171-195.

[3] V. Pestun and M. Zabzine, Introduction to localization in quantum field theory, arXiv: 1608.02953v3 [hep-th].

[4] V. Pestun, Localization of gauge theory on a four-sphere and supersymmetric Wilson loops, Commun. Math. Phys. 313 (2012) 71-129; [arXiv: 0712.2824 [hep-th]].

[5] F. Benini and S. Cremonesi, Partition Functions of $\mathcal{N}=(2,2)$ Gauge Theories on $S^{2}$ and Vortices, Commun. Math. Phys. 334 (2015) 1483-1527; [arXiv: 1206.2356 [hep-th]].

[6] N. Doroud, J. Gomis, B. Le Floch and S. Lee, Exact results in D $=2$ Supersymmetric Gauge Theories, JHEP 05 (2013) 093; [arXiv: 1206.2606 [hep-th]].

[7] A. Kapustin, B. Willett and I. Yaakov, Exact Results for Wilson Loops in Superconformal Chern-Simons Theories with Matter, JHEP 03 (2010) 089; [arXiv: 0909.4559 [hep-th]]. 
[8] B. Willett, Localization on three-dimensional manifolds, Journal of Physics A: Mathematical and Theoretical 50 (20016) 443006; [arXiv: 1608.02958v3 [hep-th]].

[9] N. A. Nekrasov, Seiberg-Witten prepotential from instanton counting, Proceedings of the International Congress of Mathematicians, Vol. III (Beijing, 2002), Higher Ed. Press, Beijing, 2002, pp. 477-495.

[10] J. Kallen and M. Zabzine, Twisted supersymmetric 5D Yang-Mills theory and contact geometry, JHEP 05 (2012) 125; [arXiv: 1202.1956 [hep-th]].

[11] H.-C. Kim and S. Kim, M5-branes from gauge theories on the 5-sphere, JHEP 05 (2013) 144; [arXiv: 1206.6339 [hep-th]].

[12] A. Narukawa, The modular properties and the integral representations of the multiple elliptic gamma functions, Advances in Mathematics, 189 (2004) 247-267.

[13] G. E. Andrews, The Theory of Partitions, Encyclopedia of Mathematics vol. 2, AddisonWesley Publishing Company, 1976.

[14] E. T. Bell, Exponential Polynomials, Ann. Math. 35 (1934) 258-277.

[15] L. Bonora and A. A. Bytsenko, Partition Functions for Quantum Gravity, Black Holes, Elliptic Genera and Lie Algebra Homologies, Nucl. Phys. B 852 (2011) 508-537.

[16] A. A. Bytsenko, M. Chaichian, R. J. Szabo and A. Tureanu, Quantum Black Holes, Elliptic Genera and Spectral Partition Functions, IJGMMP 11 (2014) 1450048; [arXiv: 1308.2177 [hep-th]].

[17] D. B. Fuks, Cohomology of Infinite-Dimensional Lie Algebras, Consultants Bureau, New York and London, 1986.

[18] G. E. Andrews, q-Series: Their Development and Application in Analysis, Number Theory, Combinatorics, Physics, and Computer Algebra, Conference Board of the Mathematical Society 66, Providence, Rhode Island 1985.

[19] F. H. Jackson, The basic gamma-function and the elliptic functions, Proc. Roy. Soc. London, A 76 (1905) 127-144.

[20] V. P. Spiridonov, Theta hypergeometric integrals, Algebra i Analiz 15 (2003) 161-215; [arXiv: 0303205v2 [math]].

[21] G. Gasper and M. Rahman, Basic Hypergeometric Series, Encyclopedia of Mathematics and its Applications, Ed. by G.-C. Rotta, Cambridge University Press 35, 1990.

[22] S. Pasquetti, Factorization of N=2 theopries on the squashed 3-sphere, JHEP 04 (2012) 120; [arXiv: 1111.6905 [hep-th]].

[23] T. Dimofte, D. Gaiotto and S. Gukov, 3-Manifolds and 3d Indices, Adv. Theor. Math. Phys (2013) 975-1076; [arXiv: 1112.5179 [hep-th]]. 
[24] C. Beem, T. Dimofte and S. Pasquetti, Holomorphic blocks in three dimensions, JHEP 12 (2014) 177; [arXiv: 1211.1986 [hep-th]].

[25] Y. Imamura, H. Matsuno and D. Yokoyama, Factorization of the $S^{3} / \mathbb{Z}_{n}$ partition function, Phys. Rev. D 89 (2014) 085003; [arXiv: 1311.2371 [hep-th]].

[26] F. Nieri and S. Pasquetti, Factorization and holomorphic blocks in 4d, JHEP 11 (2015) 155; [arXiv: 1507.00261 [hep-th]].

[27] Y. Yoshida and K. Sugiyama, Localization of $3 d \mathcal{N}=2$ Supersymmetric Theories on $S^{1} \times D^{2}$, arXiv: 14096713 [hep-th]. 